\title{
Nuclear Medicine Study File
}

National Cancer Institute

\section{Source}

National Cancer Institute. Nuclear Medicine Study File. NCI Thesaurus. Code C115502.

A collection of nuclear medicine files pertaining to a clinical study. 